\title{
Photometric Determination of the Distance to the RR Lyrae LP Cam.
}

\author{
Brian UZPEn ${ }^{1,2, *}$ AND TIMOThy F. SLATER ${ }^{2}$
}

\author{
${ }^{1}$ Natural Sciences Department, Laramie County Community College, 1400 E. College Drive, Cheyenne, WY 82007 \\ ${ }^{2}$ School of Teacher Education, College of Education, 1000 E. University Avenue, Laramie, WY 82071 \\ "Corresponding author: buzpen@gmail.com
}

\begin{abstract}
Photometric observations of the nearby RR Lyrae, LP Cam, were obtained to verify that the period-luminosity relationships are still valid for RR Lyrae stars with improved parallaxes as part of a larger study. Using both obtained photometry and archival data, photometric distance estimates were made to LP Cam. When accounting for independantly measured values of interstellar reddenning, the photometric distance determined for LP Cam is not in agreement with the parallactic distance. The likely causes for this mismatch are explored and could be due to greater extinction than measured or incorrect measured effective temperatures from spectral energy distributions. An additional cause could be due to a faint proximate source to LP Cam. When $E(B-V)$ is changed such that the variance in distance measured between the three filters is minimized, an estimate of $E(B-V) \approx 0.24$ is achieved with distance estimates of V: $780 \pm 40$ pc, i: $793 \pm 41 \mathbf{p c}, \mathbf{z :} 792 \pm 38$ pc which compares reasonably well with the GAIA value of $809 \pm 20$ pc

(수요 $\odot 2020$ Astronomy Theory, Observations and Methods Journal
\end{abstract}

Keywords: Stars: Variable: RR Lyrae, techniques: photometric, catalogs, parallaxes

http://dx.doi.org/etc

\section{INTRODUCTION}

RR Lyrae stars are low-mass horizontal branch stars with short periods of pulsations less than a day. These stars play an invaluable role in understanding the distances of our Universe and are one of several standard candles used to set the distance scale within astronomy. However, it wasn't until Longmore et al. (1986) that the period-luminosity relationship of RR Lyrae stars could be leveraged to determine extragalactic distances. With the advancements of computational stellar photospheric models, Catelan et al. (2004) and Cáceres \& Catelan (2008) were able to derive theo- retical relationships for the absolute magnitude of $\mathrm{RR}$ Lyrae stars in common broadband filters. With the increasingly precise parallax measurements of surveys, such as GAIA (Gaia Collaboration et al., 2018), it has yet to be determined whether the distances for the brightest RR Lyrae using the well established relationships of Cáceres \& Catelan (2008) and Catelan et al. (2004) are in agreement with the distances determined via $G A I A$ 's parallax measurements. The aim of this study is to obtain period relationships in $B, V$, $i$, and $z$ filters to determine the distances to the star photometrically and make sure these distances are in 
agreement with the distance from parallax measurements.

\section{OBSERVATIONS/METHODS}

We scheduled 57 cadence observations using the Las Cumbres Observatory Network between September 26-October 9, 2019 in Johnsons $B$ and $V$ and SDSS $i$ and $z$ filters. We were able to obtain 47 images during this window. These observations of the variability of LP Cam allow us to determine the period through generating lightcurves, verify the temperature and luminosity of the star using color relationships, and in combination with previously measured archival values determine the distance to the star.

\section{Image Processing}

Images were processed using the $O S S$ pipeline (Fitzgerald, 2018), which includes basic image processing such as cropping, flat fielding and cosmic ray reduction. The $O S S$ pipeline also performs photometric source extraction and both aperture photometry or point-spread photometry using algorithms such as DAOphot (Stetson, 1987). Photometric reduction algorithms were performed on all images in each of the bandpasses. The data was further analyzed via Astropy-based python scripts called astrosource that calibrates the sources through identification of the least variable stars in the field, determines their apparent magnitude and then determines the period of the RR Lyrae. With redder filters, fewer comparison stars were identified with 27 stable comparisons stars in $B, 25$ in $V, 46$ in $i$, and only 31 in $z$. Results for the calibrated apparent magnitude of the LP Cam lightcurves in each bandpass using PSF photometry are shown in Table 1.

\begin{tabular}{ccc}
\hline Filter & Mag & e(Mag) \\
\hline$B$ & 12.248 & 0.045 \\
$V$ & 11.322 & 0.044 \\
$i$ & 10.795 & 0.050 \\
$z$ & 10.661 & 0.037 \\
\hline
\end{tabular}

Table 1. Calibrated Apparent Magnitudes. The calibrated magnitudes of LP Cam from PSF photometry in each filter along with measured uncertainties.

\section{RESULTS}

\section{RR Lyrae Verification}

It is not uncommon for variable stars to be misidentified in the literature. As a check, we used $B-V$ and the estimated maximum extinction from the Dust Calculator using the method of Schlafly \& Finkbeiner (2011), to estimate the luminosity and effective temperature of the star. The estimated maximum reddenning is $E(B-V)=0.6554 \pm 0.0278$. We determined the luminosity using the relationship in Equation 1.

$$
\log \frac{L_{*}}{L_{\text {solar }}}=\frac{-(B-V)-4.77}{2.5}
$$

The effective temperature was estimated using both the non-reddenned and reddenned $(B-V)$ relationships using Equation 2 (Ballesteros, 2012).

$$
T_{\text {eff }}=\left(\frac{1}{0.92(B-V)+1.7}+\frac{1}{0.92(B-V)+0.62}\right) * 4600
$$

The results are summarized in Table 2 and show that LP Cam clearly occupies a position in the HR Diagram indicative of being an RR Lyrae star.

\begin{tabular}{cccc}
\hline & $(B-V)$ & $\log \frac{L_{*}}{L_{s} \text { olar }}$ & $\log \left(T_{\text {eff }}\right)$ \\
\hline Non-Reddened & 0.926 & 1.538 & 3.69 \\
Reddened & 0.313 & 1.783 & 3.87 \\
\hline
\end{tabular}

Table 2. Effective Temperature and Luminosity We present the maximum and minimum possible extinction values to the RR Lyrae LP Cam. Regardless of the level of reddenning, LP Cam occupies a space in the HR Diagram consistent with RR Lyrae stars.

\section{Period Determination}

The period in each of the filters was determined using both the Phase Dispersion Minimization method (PDM) (Stellingwerf, 1978) and the String-Length method (SL) (Lafler \& Kinman, 1965). The results for each of the filters are reported in Table 3 in days. The residuals of the fit using both methods are shown in Figure 1 and 2, respectively. The lightcurves for all four bandpasses are shown in Figures 3, 4, 5, and 6.

\section{DISCUSSION}

\section{Photometry}

Due to the relative brightness of LP Cam there are a multitude of observations to compare our measurements with. Photometric measurements from this 


\begin{tabular}{cccccc}
\hline Filter & $\begin{array}{c}\text { PDM } \\
\text { (days) }\end{array}$ & $\begin{array}{c}\mathrm{e} \text { (PDM) } \\
\text { (days) }\end{array}$ & $\begin{array}{c}\text { SL } \\
\text { (days) }\end{array}$ & $\begin{array}{c}\mathrm{e}(\mathrm{SL}) \\
\text { (days) }\end{array}$ & $\begin{array}{c}\text { Amp } \\
\text { (mags) }\end{array}$ \\
\hline$B$ & 0.5729 & 0.0086 & 0.5730 & 0.0093 & 0.973 \\
$V$ & 0.5729 & 0.0091 & 0.5734 & 0.0082 & 0.760 \\
$i$ & 0.5691 & 0.0100 & 0.5688 & 0.0072 & 0.489 \\
$z$ & 0.5692 & 0.0121 & 0.5771 & 0.0100 & 0.461 \\
\hline
\end{tabular}

Table 3. Period Determination. The period was determined using both the PDM and SL methods. The periods are in good agreement with each other. The amplitude of the light curve in each filter is provided in the last column.

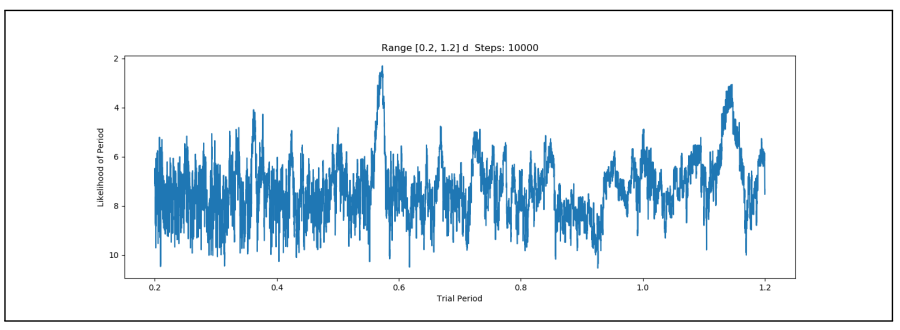

Fig. 1. The probability plot for LP Cam's phase using the String-Length method. The is a very strong likelihood of the period at just under 0.6 days with next strongest peak at double the period.

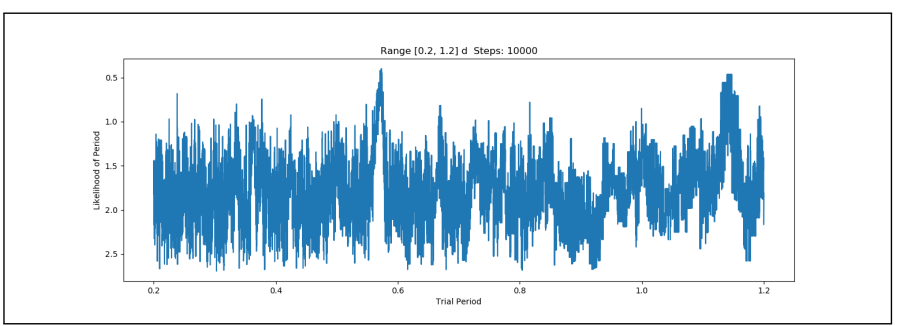

Fig. 2. The probability plot for LP Cam's phase using the Phase-dispersion minimization method. The is a very strong likelihood of the period at just under 0.6 days with next strongest peak at double the period, similar to the SL method.

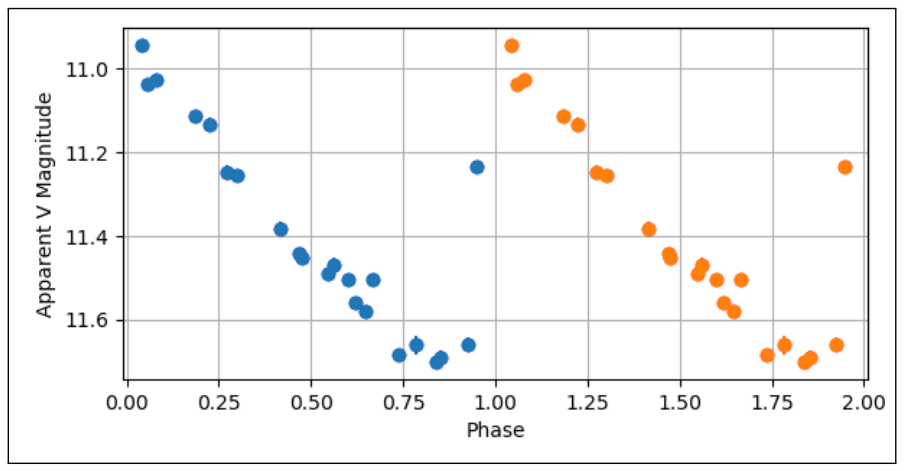

Fig. 3. A phased light curve for LP Cam in the $V$ band using the PDM period. Given that both the SL and PDM period are equivalent within uncertainties a solution using the SL period is not presented. The data are well-matched to a period of $0.5729 \pm$ 0.0091 days.

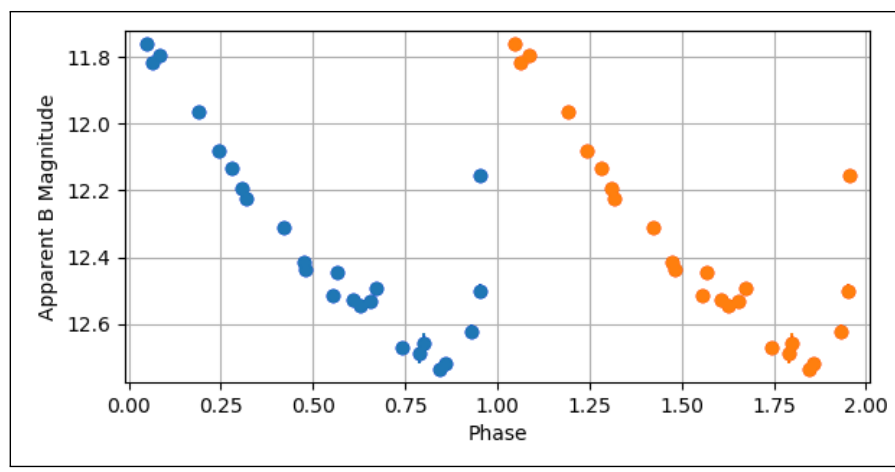

Fig. 4. A phased light curve for LP Cam in the $B$ band using the PDM period.

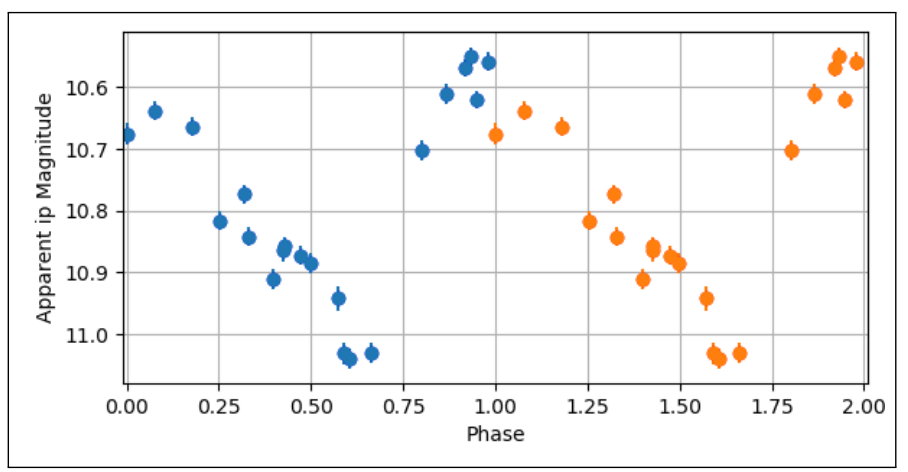

Fig. 5. A phased light curve for LP Cam in the $i$ band using the PDM period. 


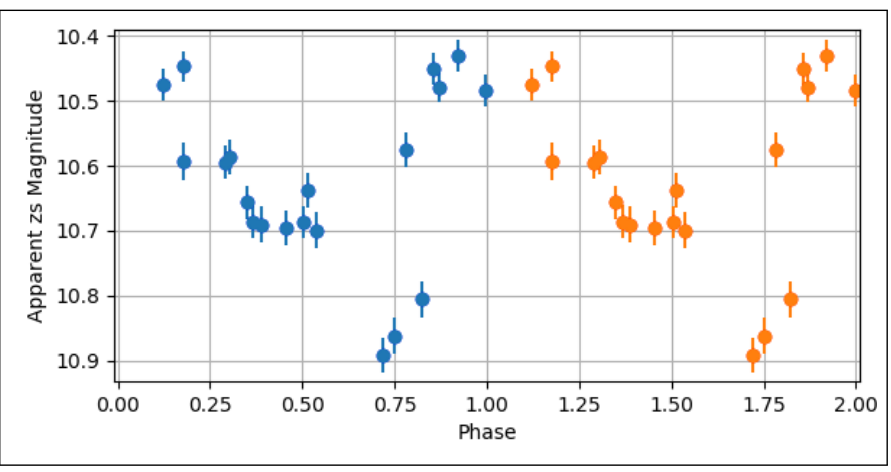

Fig. 6. A phased light curve for LP Cam in the $z$ band using the PDM period.

study are compared to literature values (with uncertainties when available) from both compiled catalogs, such as the AAVSO All-Sky Photometric Catalog (APASS, Henden et al., 2015), (All-Sky, Kharchenko, 2001), and (Panstarss, Flewelling et al., 2016) and previous studies of RR Lyraes, such as Kinemuchi et al. (2006). The results of the comparison are shown in Table 4. In general, the optical measurements of this study using PSF photometry agree with the literature values within uncertainties, with the exception of $V$ from Kinemuchi et al. (2006). The near-infrared measurements appear to be systematically brighter at $i$ than those in the literature. Upon inspection of the literature, it was found that Flewelling et al. (2016) detected a faint source within one arcsecond in $r$ and $i$ bands. As a test, aperture photometry was compared to the literature values and the aperture values agree better with those previously detected. However, given the proximate nature of this faint source, PSF photometry would be the correct approach to minimize errors for stars in a crowded field and we therefore adopt the values determined from PSF photometry for the remaining analysis (e.g., Janes \& Heasley, 1993). It is important to note that the faint source was not detected in the $g$ and $z$ filters in the Panstarrs data set and is not detected in our relatively short exposures of LP Cam using the LCO $0.4 \mathrm{~m}$ telescopes.

\section{Period}

Using all four filters, the mean period was found to be $0.5716 \pm 0.0050$ days using the PDM method and $0.5731 \pm 0.0043$ days using the SL method. Both values are consistent with one-another. A comparison of the results for the period of pulsation is shown in Table 5 and our values agree with those in the literature with the exception of the mid-infrared study done by
Gavrilchenko et al. (2014). The difference between the optical and near-infrared measurements may be a result of the decrease in amplitude as the wavelength increases for these types of stars. However, without reported uncertainties it is difficult to determine if this period is truly different than the other reported values.

\section{Distance}

The purpose of this study is to determine if periodluminosity relationships from Catelan et al. (2004) Cáceres \& Catelan (2008) agree with GAIA DR2 parallax measurements for the brightest RR Lyrae. To determine the photometric distance to this star we used the measured PSF photometric values in $V, i$, and $z$, the metallicity of the star from Fourier coefficients of 0.03 Gaia Collaboration et al. (2018) and an estimated reddenning value. As previously stated, the maximum assumed reddenning is $\mathrm{E}(B-V)=0.6554$. The results for each of the filters is provided in Table 6. This maximum value provides a minimum distance of $517 \pm 16 \mathrm{pc}$ and a maximum distance of $1013 \pm 32$ pc. However, a better estimate of reddenning can be made by passing photometric measurements through a spectral energy distribution (SED) and determining the best fit. Pickles \& Depagne (2010) performed this measurement and determined that LP Cam is best fit by a G5III spectrum, which corresponds to a $\mathrm{T}_{e f f} \simeq 5010 \mathrm{~K}$ and $\mathrm{E}(B-V)=0.04$. McDonald et al. (2017) also measured $\mathrm{T}_{\text {eff }}$ using archival data and stellar atmospheric models at 5036 $\pm 182 \mathrm{~K}$, which corresponds to about 0.07 magnitudes of uncertainty. The resulting distance measurement using SED fitting for extinction and the relationships of Catelan et al. (2004) and Cáceres \& Catelan (2008) is 971士 57 pc. The measured distance from Gaia Collaboration et al. (2018) is $809 \pm 20$ pc. These two derived measurements are not in agreement. In order for our photometric measurements to match this distance, the reddenning to LP Cam must $\mathrm{E}(B-V) \simeq$ 0.24 magnitudes. Using $\mathrm{E}(B-V)=0.24$, we find that the distance using each individual bandpass and their weighted average all overlap with the measured $G A I A$ distance with variance between the photometric data and parallactice data minimized. Thus, we adopt an $\mathrm{E}(B-V)$ value of 0.24 for LP Cam. Furthermore, it is important to note that Gavrilchenko et al. (2014) using mid-infrared data determined a distance of $843 \pm 14$ pc for the distance to LP Cam, which is in agreement with the GAIA measured dis- 


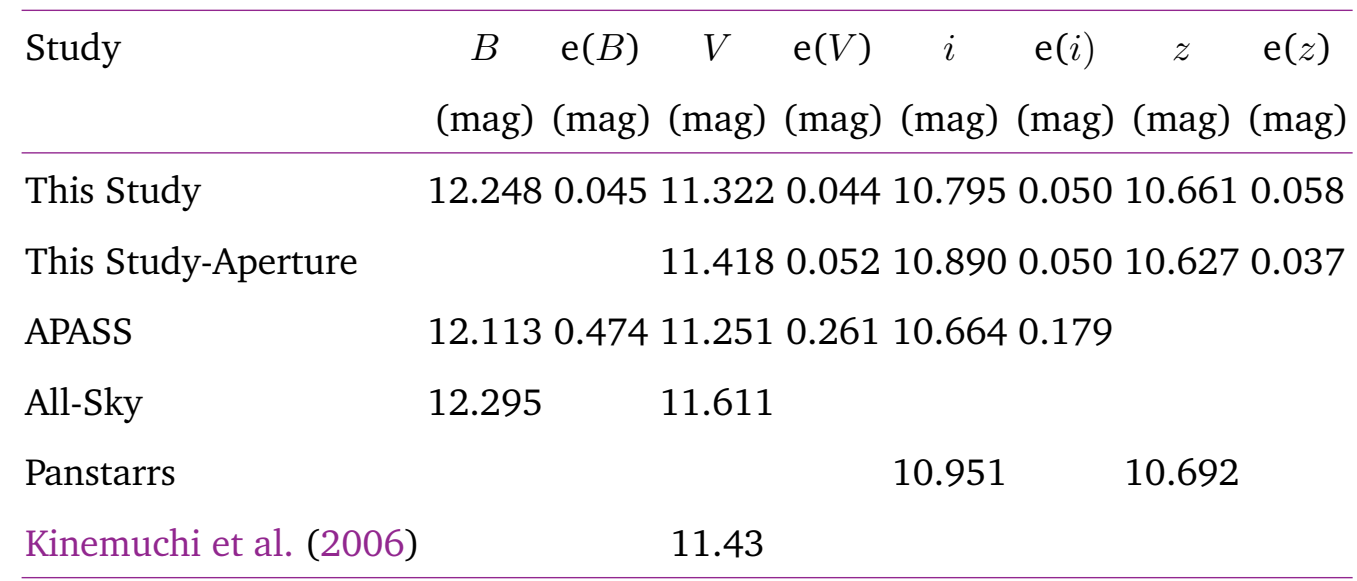

Table 4. Photometry Comparison. The calibrated measured photometry from this study compared to values within the literature. Within uncertainties, the values from this study agree with those from the literature using aperture photomery. However, PSF photometry, which we argue is the correct approach, is noticeably brighter in $V$ and $i$ than Panstarrs, All-Sky and Kinemuchi et al. (2006).

\begin{tabular}{lcc}
\hline Study & $\begin{array}{c}\text { Period } \\
\text { (days) }\end{array}$ & $\begin{array}{c}\text { e(Period) } \\
\text { (days) }\end{array}$ \\
\hline This study & 0.5716 & 0.0050 \\
Watson et al. (2006) & 0.5720300000 & \\
Kinemuchi et al. (2006) & 0.57205 & \\
Maintz (2005) & 0.572092000 & \\
Gavrilchenko et al. (2014) & 0.5840 & \\
\hline
\end{tabular}

Table 5. Period Comparison. The period of pulsation from this study is compared to those of previous studies. In general, there is good agreement between the measured values of this study and those of previous studies. The exception is the midinfrared period of Gavrilchenko et al. (2014). The difference in observed period may be due to the decrease in amplitude as the wavelength increases for these types of stars. tance and extinction decreases with increasing wavelength bolstering the argument to adjust $\mathrm{E}(B-V)$ from the maximum value. Also of note, is that if this analysis were repeated using aperture instead of PSF photometry, the measured distance would increase slightly and still not be in agreement with the measured parallactic distance using redenning values determined from $\mathrm{T}_{\text {(eff }}$ ) archival data or maximum interstellar redenning. Aperture photometry would require an even greater extinction in order to agree with the parallactic distance than our adopted value of $\mathrm{E}(B-V)=0.24$.

\section{CONCLUSION AND IMPLICATIONS}

Using the relationships of Catelan et al. (2004) and Cáceres \& Catelan (2008), the minimum distance to LP Cam is $517 \pm 16$ pc with a maximum distance of $1013 \pm 32$ pc. These distances are highly dependent upon interstellar reddenning values. Using previously determined values of interstellar redenning of $\mathrm{E}(B-V)=0.04$ from SED fitting (Pickles \& Depagne, 2010), we find the mean distance of LP Cam using all four bandpasses to be $971 \pm 57$ pc. This measured distance is not in agreement with measured parallax from Gaia Collaboration et al. (2018). The difference between these measurements could be due to errors in archival photometric measurements for this source. LP Cam has a faint proximate source that has been detected in some of the filters in the Panstarrs dataset. Depending upon the type of photometric extraction of the data, the light from this star could be affecting short exposures of LP Cam and subsequent SED fit- 


\begin{tabular}{cccccccccc}
\hline $\mathrm{E}(B-V)$ & $V$ & $\mathrm{e}(V)$ & $i$ & $\mathrm{e}(i)$ & $z$ & $\mathrm{e}(z)$ Mean e(Mean) \\
\hline 0.6554 & 431 & 27 & 529 & 27 & 592 & 28 & 517 & 16 \\
0 & 1099 & 68 & 1002 & 52 & 938 & 44 & 1013 & 32 \\
0.24 & 780 & 48 & 793 & 41 & 792 & 38 & 788 & 24 \\
0.04 & 1038 & 133 & 963 & 92 & 912 & 72 & 971 & 57 \\
\hline
\end{tabular}

Table 6. Distance Comparison. Using the relationships of Catelan et al. (2004) \& Cáceres \& Catelan (2008), the minimum average photometric distance to the star is $517 \pm 16 \mathrm{pc}$, while the maximum distance is $1013 \pm 32$ pc. Neither of these measurements are in good agreement with the parallactic measurement from GAIA DR2 of $809 \pm 20$ pc. Using the synthetically derived $\mathrm{T}_{\text {eff }}$ measured from McDonald et al. (2017) \& Pickles \& Depagne (2010) the distance to LP Cam is overestimated when comparing to the distance from $G A I A$.

ting that was used to estimate a reasonable value for interstellar extinction. Spectroscopic measurements should be conducted of LP Cam to determine the temperature (and subsequent) extinction to LP Cam. An interstellar redenning value of $\mathrm{E}(B-V)=0.024$ would result in the distances determined using periodluminosity relationships of Catelan et al. (2004) and Cáceres \& Catelan (2008) agreeing with the measured GAIA values of distance for LP Cam with a mean derived photometric distance of $788 \pm 24$ pc. Furthermore, the faint source proximate to LP Cam should be investigated for the possibility of binarity. Few RR Lyraes reside in binary systems (e.g., Hajdu et al., 2015) and this object presents a rare opportunity to determine key stellar parameters (such as mass) for a unique proximate standard candle.

\section{REFERENCES}

Ballesteros, F. J. (2012, feb). New insights into black bodies. EPL (Europhysics Letters), 97(3), 34008. Retrieved from https://doi.org/10.1209/0295 -5075/97/34008 doi:

Cáceres, C., \& Catelan, M. (2008). The periodluminosity relation of rr lyrae stars in the sdss photometric system. The Astrophysical Journal Supplement Series, 179(1), 242. Retrieved from https://doi.org/10.1086/591231
Catelan, M., Pritzl, B. J., \& Smith, H. A. (2004). The rr lyrae period-luminosity relation. i. theoretical calibration. The Astrophysical Journal Supplement Series, 154(2), 633. Retrieved from https://doi . org/10.1086/422916

Fitzgerald, M. T. (2018). The our solar siblings pipeline: Tackling the data issues of the scaling problem for robotic telescope based astronomy education projects. RTSRE, 1(1), 347-358.

Flewelling, H. A., Magnier, E. A., Chambers, K. C., Heasley, J. N., Holmberg, C., Huber, M. E., ... Shiao, B. (2016, Dec). The Pan-STARRS1 Database and Data Products. arXiv e-prints, arXiv:1612.05243.

Gaia Collaboration, Brown, A. G. A., Vallenari, A., Prusti, T., de Bruijne, J. H. J., Babusiaux, C., ... Zwitter, T. (2018, Aug). Gaia Data Release 2. Summary of the contents and survey properties. , 616, A1. Retrieved from https://doi.org/10 .1051/0004-6361/201833051

Gavrilchenko, T., Klein, C. R., Bloom, J. S., \& Richards, J. W. (2014, Jun). A mid-infrared study of RR Lyrae stars with the Wide-field Infrared Survey Explorer all-sky data release. , 441(1), 715725. Retrieved from https://doi.org/10.1093/ mnras/stu606

Hajdu, G., Catelan, M., Jurcsik, J., Dékány, I., Drake, A. J., \& Marquette, J.-B. (2015, 03). New RR Lyrae variables in binary systems. Monthly Notices of the Royal Astronomical Society: Letters, 449(1), L113L117. Retrieved from https://doi.org/10.1093/ mnrasl/slv024

Henden, A. A., Levine, S., Terrell, D., \& Welch, D. L. (2015, Jan). APASS - The Latest Data Release. In American astronomical society meeting abstracts \#225 (Vol. 225, p. 336.16).

Janes, K. A., \& Heasley, J. (1993). Stellar photometry software. Publications of the Astronomical Society of the Pacific, 105(687), 527. Retrieved from https://doi.org/10.1086/133187

Kharchenko, N. V. (2001, Oct). All-sky compiled catalogue of 2.5 million stars. Kinematika i Fizika Nebesnykh Tel, 17(5), 409-423. 
Kinemuchi, K., Smith, H. A., Woźniak, P. R., McKay, T. A., \& ROTSE Collaboration. (2006, Sep). Analysis of RR Lyrae Stars in the Northern Sky Variability Survey. , 132(3), 1202-1220. Retrieved from https://doi.org/10.1086/506198

Lafler, J., \& Kinman, T. D. (1965, Jun). An RR Lyrae Star Survey with the Lick 20-INCH Astrograph II. The Calculation of RR Lyrae Periods by Electronic Computer. , 11, 216. Retrieved from https://doi . org/10.1086/190116

Longmore, A., Fernley, J., \& Jameson, R. (1986). Rr lyrae stars in globular clusters: better distances from infrared measurements? Monthly Notices of the Royal Astronomical Society, 220(2), 279287. Retrieved from https://doi.org/10.1093/ mnras/220.2.279

Maintz, G. (2005, Oct). Proper identification of RR Lyrae stars brighter than 12.5 mag. , 442(1), 381384. Retrieved from https://doi.org/10.1051/ 0004-6361:20053230

McDonald, I., Zijlstra, A. A., \& Watson, R. A. (2017, Oct). Fundamental parameters and infrared excesses of Tycho-Gaia stars. , 471(1), 770-791. Retrieved from https://doi.org/10.1093/mnras/ stx1433

Pickles, A., \& Depagne, É. (2010, Dec). All-Sky Spectrally Matched UBVRI - ZY and u g r i z Magnitudes for Stars in the Tycho2 Catalog. , 122(898), 1437. Retrieved from https://doi.org/10.1086/ 657947

Schlafly, E. F., \& Finkbeiner, D. P. (2011). Measuring reddening with sloan digital sky survey stellar spectra and recalibrating sfd. The Astrophysical Journal, 737(2), 103. Retrieved from https://doi.org/ $10.1088 / 0004-637 X / 737 / 2 / 103$

Stellingwerf, R. F. (1978, Sep). Period determination using phase dispersion minimization. , 224, 953960. Retrieved from https://doi.org/10.1086/ 156444

Stetson, P. B. (1987). Daophot: A computer program for crowded-field stellar photometry. Publications of the Astronomical Society of the Pacific, 99(613), 191. Retrieved from https://doi.org/10.1086/ 131977
Watson, C. L., Henden, A. A., \& Price, A. (2006, May). The International Variable Star Index (VSX). Society for Astronomical Sciences Annual Symposium, $25,47$. 